\title{
Found Dead
}

National Cancer Institute

\section{Source}

National Cancer Institute. Found Dead. NCI Thesaurus. Code C90387.

An indication that a subject was found in a deceased state. 